\title{
PARTICIPACIÓN CIUDADANA EN LOS PLANES DE DESARROLLO Y ORDENAMIENTO TERRITORIAL DE LA CIUDAD DE IBARRA EN ECUADOR
}

\author{
Giovanny Darío Guerrero Quimbiulco ${ }^{1 *}$, Jorge Patricio Romero Galarza ${ }^{1}$ \\ ${ }^{1}$ Pontificia Universidad Católica del Ecuador Sede Ibarra-Escuela de Arquitectura \\ *Autor para correspondencia: gdguerrero@pucesi.edu.ec \\ Recibido: 2021/03/08 Aprobado: 2021/06/04 \\ D0l: https://doi.org/10.26621/ra.v1i24.668
}

\section{RESUMEN}

Para las grandes democracias, la participación directa de la ciudadanía en la gobernanza plantea un desafío debido a la implementación de mecanismos como los "consejos ciudadanos", los "consejos consultivos", los "presupuestos participativos" entre otros, para que los ciudadanos formen parte integrante en el sistema de gobierno, compartiendo responsabilidades de elaborar los planes locales de acción. Si bien es cierto que en Ecuador se garantiza la representatividad participativa, estableciendo normas que promueven la participación ciudadana en los asuntos públicos, todavía existen vacíos que impiden un desarrollo de la intervención ciudadana en varios gobiernos locales, como es el caso del GAD (Gobierno Autónomo Descentralizado) del cantón Ibarra. Por esta razón y con el propósito de conocer el contexto en el que se desenvuelven los procesos participativos en este GAD, se estableció la necesidad de aplicar técnicas de recopilación de información cualitativa como la entrevista, que fue dirigida a siete funcionarios que colaboran con los procesos de participación ciudadana a nivel de desarrollo territorial; y cuantitativa, como la encuesta dirigida a veinticuatro representantes de las cinco parroquias urbanas y siete rurales de lbarra. Los resultados de estas entrevistas y encuestas mostraron que el compromiso ciudadano presenta muchas limitaciones relacionadas con la falta de comunicación, la baja capacitación, la carencia de presentación de los resultados de las mesas de trabajo a la ciudadanía permanentemente. Asimismo, existen otras barreras que surgen del limitado presupuesto, la falta de decisión política y el análisis y evaluación de resultados, que basados en indicadores, promuevan mecanismos eficientes de participación ciudadana; fundamentados en la capacidad de actuar, deliberar y participar con reuniones plenarias, educación ciudadana y capacitación a funcionarios del GAD, construyendo alianzas con los ciudadanos y controlando y evaluando la participación ciudadana para una toma de decisiones eficiente a nivel de gestión local.

Palabras clave: participación ciudadana, planificación territorial, desarrollo local, gobernanza, mecanismos participativos.

\section{ABSTRACT}

For large democracies, the direct participation of citizens in governance, mainly local, poses a great challenge due to the implementation of mechanisms such as "citizen councils", "consulta-tive councils", "participatory budgets" among others, so that citizens can be an integral part of the government system, sharing with them the responsibilities of drawing up local action plans. Although it is true that in Ecuador participatory representativeness is guaranteed, establishing norms that promote citizen participation in public affairs, there are still certain gaps that prevent an adequate development of citizen intervention in several local governments, as is the case of the GAD of the canton Ibarra. For this reason and with the purpose of knowing the context in which the participatory processes unfolds in the Ibarra's GAD, the need to apply qualitative information gathering techniques such as the interview was established, which was directed to seven officials who collaborate with the citizen participation processes at the territorial develop-ment level; and quantitative, such as the survey directed to twenty-four representatives of the five urban and seven rural parishes of the canton Ibarra. The results of these interviews and sur-veys showed that citizen engagement still has many limitations related to the lack of communica-tion, low training, and the lack of permanent presentation of the results of the work tables to the citizens. Likewise, there are other barriers that arise from the limited budget, the lack of political decision and the analysis and evaluation of results, which, based on indicators, can promote more efficient mechanisms of citizen participation; These mechanisms should be based on the ability to act, deliberate and participate permanently with plenary meetings, citizen education and training for GAD officials, building alliances with citizens and trust, as well as controlling and evaluating citizen participation processes for more efficient decision-making at the local man-agement level.

Keywords: citizen participation, territorial planning, local development, governance, participatory mechanisms.

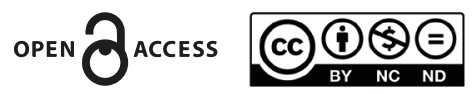




\section{INTRODUCCIÓN}

Desde la aprobación de la Constitución del año 2008, Ecuador ha creado varias innovaciones democráticas importantes; además, el gobierno ecuatoriano ha aprobado marcos legislativos y reglamentarios que reconocen el ejercicio de los derechos de participación ciudadana en todos los aspectos de la gobernanza y la formulación de políticas públicas, donde se prevé la convocatoria de la Asamblea de Ciudadanos Plurinacionales e Interculturales para el Buen Vivir como foro de consulta y diálogo directo entre el Estado y sus ciudadanos para formular, aprobar y monitorear el Plan Nacional de Desarrollo (Acosta, 2017).

Además, la Carta Magna aborda la importancia de las organizaciones sociales como instancias esenciales de la participación ciudadana. Así, en el año 2013, se promulgó el Reglamento para el Funcionamiento del Sistema Unificado de Información de las Organizaciones Sociales, que regula el reconocimiento de las organizaciones sociales, su régimen de funcionamiento, registro, control y motivos de disolución. Posteriormente, se emite el decreto ejecutivo 739 que brinda la personalidad jurídica a las organizaciones sociales (Guillen et al., 2018).

La participación ciudadana es un proceso basado en la comunidad, donde los ciudadanos se organizan por sí mismos y sus objetivos a nivel de base; trabajan juntos a través de organizaciones comunitarias no gubernamentales para influir en la toma de decisiones (SENPLADES, 2018). Los ciudadanos se involucran más en este proceso, cuando el problema en cuestión se relaciona directamente con ellos. Además, la participación ciudadana ocurre cuando todas las partes interesadas cooperan para implementar cambios a nivel local.

El país ha implementado una normativa importante para el desarrollo de los procesos de participación ciudadana y se ha tenido una renovación completa en el ámbito institucional, que abrió las puertas para que el ciudadano común y las organizaciones sociales puedan participar activamente en los procesos democráticos, a través de los Gobiernos Autónomos Descentralizados (GAD Ibarra, 2017).

La participación ciudadana a nivel local está establecida como un derecho desde el punto de vista aplicativo en los Planes de Ordenamiento Territorial (PDOTs), pero aún es incipiente debido principalmente a que no existen procedimientos adecuados que estimulen de manera activa, que promuevan una coherencia entre las necesidades y los objetivos del plan. Esto se puede lograr a través de apoyo técnico y capacitación en el proceso de formulación, seguimiento y aprobación de los planes que garantizan la toma de decisiones de forma compartida entre la ciudadanía y el gobierno. Estos procesos no se han consolidado completamente; algunos muestran deficiencias, incumplimiento de la normativa, 0 simplemente no ha existido la voluntad política de las autoridades en aplicar adecuadamente la participación ciudadana en sus territorios.

Esta investigación nace de la inquietud y deseos de profundizar el conocimiento de la participación ciudadana en los planes de ordenamiento territorial. Además, se pretende investigar la participación ciudadana a través del monitoreo de las acciones y técnicas utilizadas en el proceso de participación, con el fin de identificar cómo se están llevando a cabo estos mecanismos y a partir de ese diagnóstico poder implementar los ajustes que sean necesarios.

El estudio aborda un análisis de la baja participación ciudadana en la elaboración de los PDOTs, en las relaciones gobierno-ciudadanía, en el involucramiento de los actores locales en la elaboración de los planes, en el grado de compromiso de las autoridades, en la participación ciudadana en el ciclo de la política pública y en las dificultades y logros del proceso participativo.

La investigación tiene como objetivo describir el marco institucional de la participación ciudadana, identificar los procesos de participación, en la medida que éstos contribuyan a dar respuestas concretas a las demandas ciudadanas, para que se pueda promover el interés por participar en la formulación del Plan de Desarrollo y Ordenamiento Territorial del Gobierno Autónomo Descentralizado (GAD) (Guerrero Quimbiulco, 2019). Además, se busca proporcionar lineamientos que estimulen la participación ciudadana en el ámbito local de la planificación territorial, y finalmente evaluar la participación en la elaboración del PDOT.

\section{MÉTODOS}

La metodología empleada para la elaboración de este estudio se estructura en cuatro fases, diferenciadas de la siguiente manera:

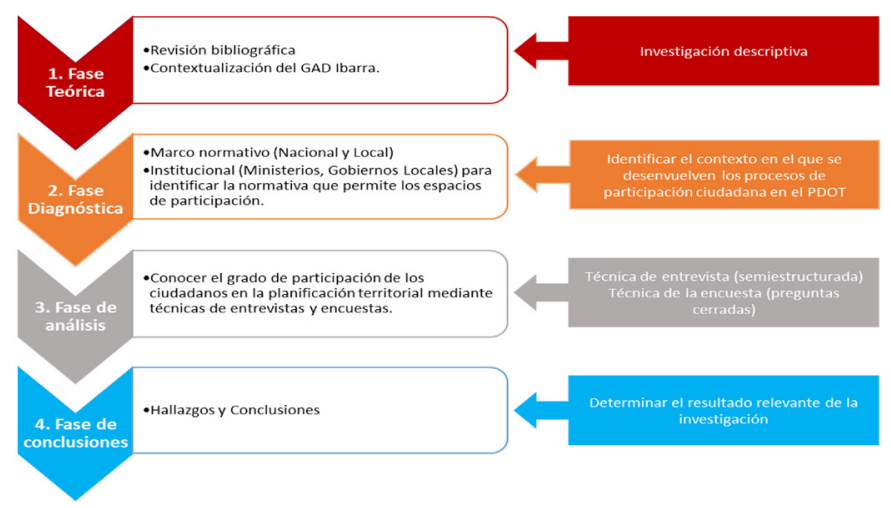

Figura 1. Metodología de la investigación

1 Fase Teórica: para el desarrollo de la fase teórica se aborda una investigación de carácter descriptiva. El estudio en lo fundamental muestra las particularidades teóricas sobre los procesos de participación ciudadana (Álvarez \& Sierra, 2012). La investigación bibliográfica se desarrolla a través de la revisión de información secundaria disponible sobre temas relacionados con la participación. En este sentido, las principales fuentes son primarias.

Los procesos participativos se han implementado durante años mediante diversas estrategias como la formulación de agendas, planes, presupuestos, observatorios, supervisión, etc. Se han desarrollado también herramientas para facilitar y organizar la participación con la inclusión de enfoques específicos, como el de género, ambiental y generacional (Calvo et al., 2017). Este grupo de herramientas, correctamente articuladas, sería parte de lo que se define como metodología participativa.

En otros países, el requisito legal para implementar los consejos locales participativos provienen de las leyes y regulaciones nacionales, que obligan a los gobiernos locales a promover la participación ciudadana y crear contextos favorables para la creación de consejos locales (Veneziano, 2017).

En el contexto de la gobernanza territorial se analiza la participación ciudadana en la elaboración de los PDOTs. La participación, si bien es cierto es reconocida bajo el sistema democrático y legal, en la práctica los mecanismos y procedimientos no son suficientes para que se hagan efectivos estos procesos; la aplicación de estas normas no garantiza la participación de los actores en la toma de decisiones para su desarrollo. 
Por otro lado, en los procesos participativos es necesario crear espacios de coordinación y oportunidades para generar sinergias y corresponsabilidades entre el sector público y privado, mantener alianzas entre el GAD y la sociedad civil que permitan desarrollar formas de participación efectivas e innovadoras, que fortalezcan a la ciudadanía para lograr incidir en una gestión pública más incluyente y participativa, que apunten dentro de un marco de equidad social, económica y de derechos, hacia una construcción e involucramiento real en la elaboración de los planes.

2 Fase Diagnóstica: se busca realizar un dictamen sobre los procesos de intervención ciudadana a nivel local, que permita identificar el contexto en el que se desenvuelven las diferentes fases y procesos de participación ciudadana en el Plan de Desarrollo y Ordenamiento Territorial (PDOT) del Gobierno Autónomo Descentralizado de Ibarra, en los siguientes aspectos:

- Marco normativo: nacional y local (constitución, decretos, leyes, acuerdos, etc.)

- Marco institucional: nacional y local (ministerios, gobiernos locales, instituciones públicas, etc.)

- Experiencias en planes territoriales: procesos de participación en los planes territoriales.

3 Fase de Análisis: La investigación tiene un enfoque mixto, haciendo uso de la triangulación metodológica, lo que ha permitido realizar una investigación que implica la recopilación, análisis e integración de resultados tanto cualitativos como cuantitativos (Hernández et al., 2014). Los datos cualitativos consisten en información abierta que presenta una diversidad de ideas recopiladas durante la aplicación de la entrevista semiestructurada, mientras que los datos cuantitativos se analizan estadísticamente con base a los puntajes obtenidos de los instrumentos (encuesta) para responder las preguntas de investigación (Albert, 2013).

La población, como un conjunto de individuos de la misma clase, limitada por el estudio, se considera al número de funcionarios del GAD Ibarra de la Comisión de Participación Ciudadana y Control Social, 2 a nivel legislativo; y de la Dirección de Planificación, Desarrollo y Ordenación Territorial, 5 a nivel ejecutivo, llegando a un total de 7 (Tabla 1).

Tabla 1. Distribución de la población para aplicación de la entrevista

\begin{tabular}{|c|c|c|c|c|}
\hline Nro. & $\begin{array}{l}\text { Parroquia } \\
\text { / Institu- } \\
\text { ción }\end{array}$ & Tipo & $\begin{array}{l}\text { Número de } \\
\text { representantes }\end{array}$ & Género \\
\hline 1 & GAD Ibarra & $\begin{array}{l}\text { Entidad Pública } \\
\text { Local Legisla- } \\
\text { tiva }\end{array}$ & 2 & 2 hombres \\
\hline 2 & GAD Ibarra & $\begin{array}{l}\text { Entidad Pública } \\
\text { Local Ejecutiva }\end{array}$ & 5 & 4 hombres 1 mujer \\
\hline Total & & & 7 & 6 hombres, 1 mujer \\
\hline
\end{tabular}

Del total de representantes de la ciudadanía, si fuese el caso líderes comunitarios, con equidad de género (masculino, femenino) de acuerdo con el tipo de representatividad, cuyas edades comprendan entre los 30 y 65 años de edad, de las 5 parroquias urbanas y las 7 rurales del cantón Ibarra, se identifican en la Tabla 2.
Tabla 2. Distribución de la población para aplicación de la encuesta

\begin{tabular}{cllcl}
\hline Nro. & Parroquia & Tipo & Representantes & \multicolumn{1}{c}{ Género } \\
\hline 1 & San Francisco & Urbana & 2 & 2 hombres \\
\hline 2 & El Sagrario & Urbana & 2 & 1 hombre 1 mujer \\
\hline 3 & Caranqui & Urbana & 2 & 1 hombre 1 mujer \\
\hline 4 & Alpachaca & Urbana & 2 & 1 hombre 1 mujer \\
\hline 5 & Priorato & Urbana & 2 & 2 mujeres \\
\hline 6 & San Antonio & Rural & 2 & 2 hombres \\
\hline 7 & La Esperanza & Rural & 2 & 2 hombres \\
\hline 8 & Angochagua & Rural & 2 & 1 hombre 1 mujer \\
\hline 9 & Ambuquí & Rural & 2 & 1 hombre 1 mujer \\
\hline 10 & Salinas & Rural & 2 & 1 hombre 1 mujer \\
\hline 11 & La Carolina & Rural & 2 & 2 mujeres \\
\hline 12 & Lita & Rural & 2 & 2 mujeres \\
\hline Total & & & 24 & 12 hombres, \\
& & & & 12 mujeres \\
\hline
\end{tabular}

El total del universo consultado en la entrevista son las personas del GAD involucradas en el área de participación social, tanto a nivel directivo como legislativo, como se muestra en la tabla número 1. Por otro lado, los líderes del cantón que representan a los 181.175 habitantes del territorio son un total de 24, que se describen en la tabla número 2.

La aplicación en sí del instrumento de investigación de tipo cuantitativo, como es la encuesta, incluye la elaboración de un cuestionario, para recopilar la información de forma organizada, de acuerdo con las variables identificadas en la investigación (Hernández et al., 2014), que se realiza con base a la operacionalización de las variables identificadas para el proceso investigativo, el concepto, los indicadores e instrumentos, de la siguiente manera indicada en la Tabla 3.

4 Fase de Conclusiones: Basada en los principales hallazgos encontrados en el tema investigado, se determinan las principales conclusiones del estudio.

\section{RESULTADOS Y DISCUSIÓN}

Con el propósito de conocer en qué contexto se desenvuelve actualmente la participación ciudadana en el PDOT del cantón Ibarra, se aplicó una entrevista que midió el grado de involucramiento de los actores que demandan de las autoridades, puntos de vista sobre la problemática del cantón, el nivel de preocupación del proceso participativo, el grado de compromiso de la ciudadanía en el proceso y el reconocimiento de los sistemas de veedurías sociales. La entrevista se realizó a siete funcionarios del GAD municipal de Ibarra: dos de la Comisión de Participación Ciudadana y Control Social (nivel legislativo), y cinco de la Dirección de Planificación, Desarrollo y Ordenación Territorial (nivel ejecutivo), (SENPLADES, 2017) cuyos resultados más importantes se muestran a continuación:

\section{Entrevista}

En relación con los mecanismos utilizados en la participación ciudadana en el GAD Ibarra, un funcionario ejecutivo menciona que estos se encuentran establecidos en la Constitución, las leyes y guías metodológicas que existen para el efecto (GAD, Ibarra, 2011); sin embargo, indica también que es necesario fomentarlos y cumplirlos. Considera que se han creado espacios de participación, aunque en ocasiones las personas no participan 
debido a su desconocimiento, demostrando una actitud apática y falta de compromiso en cuanto a sus deberes y obligaciones como ciudadanos.

Tabla 3. Operacionalización de las variables

\begin{tabular}{|c|c|c|c|}
\hline Variable & Definición conceptual & Indicadores & Instrumento \\
\hline \multirow{7}{*}{$\begin{array}{l}\text { Participa- } \\
\text { ción ciu- } \\
\text { dadana }\end{array}$} & \multirow{7}{*}{$\begin{array}{l}\text { Proceso mediante el } \\
\text { cual los miembros de } \\
\text { una sociedad (ciuda- } \\
\text { danía) comparten el } \\
\text { poder con funciona- } \\
\text { rios públicos al tomar } \\
\text { decisiones importan- } \\
\text { tes relacionadas con } \\
\text { la comunidad }\end{array}$} & $\begin{array}{l}\text { Porcentaje de progra- } \\
\text { mas y proyectos }\end{array}$ & Cuestionario \\
\hline & & $\begin{array}{l}\text { Nivel para conocer las } \\
\text { necesidades de la co- } \\
\text { munidad }\end{array}$ & Cuestionario \\
\hline & & $\begin{array}{l}\text { Nivel de participación } \\
\text { del GAD }\end{array}$ & Cuestionario \\
\hline & & $\begin{array}{l}\text { Nivel de recopilación de } \\
\text { demandas y preocupa- } \\
\text { ciones para ser inclui- } \\
\text { dos en la preparación, } \\
\text { ejecución y evaluación } \\
\text { de los PDOT }\end{array}$ & Cuestionario \\
\hline & & $\begin{array}{l}\text { Nivel de participación } \\
\text { del Ejecutivo y Legislati- } \\
\text { vo de los resultados de } \\
\text { las mesas de trabajo en } \\
\text { que participaron repre- } \\
\text { sentantes de la ciuda- } \\
\text { danía }\end{array}$ & Cuestionario \\
\hline & & $\begin{array}{l}\text { Nivel de eficiencia de } \\
\text { los mecanismos de in- } \\
\text { formación sobre el pro- } \\
\text { greso de los PDOT del } \\
\text { cantón }\end{array}$ & Cuestionario \\
\hline & & $\begin{array}{l}\text { Nivel de mejoramien- } \\
\text { to de los procesos de } \\
\text { participación ciudada- } \\
\text { na, para la elaboración, } \\
\text { ejecución y evaluación } \\
\text { de los PDOT del cantón }\end{array}$ & Cuestionario \\
\hline & $\begin{array}{l}\text { Proceso de toma de } \\
\text { decisiones encami- } \\
\text { nado a alcanzar ob- } \\
\text { jetivos económicos, }\end{array}$ & $\begin{array}{l}\text { Nivel participación y } \\
\text { presentación para la } \\
\text { elaboración de los Pla- } \\
\text { nes de Desarrollo }\end{array}$ & Cuestionario \\
\hline $\begin{array}{l}\text { PI anes } \\
\text { de De- } \\
\text { sarrollo } \\
\text { y Orde- } \\
\text { namiento } \\
\text { Territorial }\end{array}$ & $\begin{array}{l}\text { culturales, sociales y } \\
\text { ambientales mediante } \\
\text { estrategias, visiones, } \\
\text { planes espaciales y } \\
\text { la aplicación de un } \\
\text { conjunto de principios } \\
\text { de políticas, herra- } \\
\text { mientas, mecanismos } \\
\text { institucionales y parti- } \\
\text { cipativos. }\end{array}$ & $\begin{array}{l}\text { Nivel de preparación, in- } \\
\text { formación, capacitación } \\
0 \text { asesoramiento para } \\
\text { la participación en la } \\
\text { elaboración, ejecución y } \\
\text { evaluación de PDOT del } \\
\text { cantón }\end{array}$ & Cuestionario \\
\hline
\end{tabular}

Respecto a determinar los problemas principales del GAD de lbarra que se deben tomar en cuenta en la elaboración de los planes, todos los actores concuerdan que estos son: el saneamiento ambiental, el componente económico productivo que permite generar fuentes de empleo, el componente sociocultural para tratar temas como la inseguridad, la salud y la educación, entre otros.

Con respeto a generar espacios de participación desde el GAD Ibarra, uno de los encargados del Departamento de Participación Ciudadana explica: "la gestión de la participación desde municipalidad fomenta espacios de socialización e intercambio con organizaciones sociales, comités, juntas parroquiales y ciudadanía a través de convocatorias para conocer lo que sucede en el territorio". Como parte del proceso de esta participación, se establecen mesas de trabajo temáticas, relacionadas con decisiones sobre las distintas problemáticas del territorio, cuyos resultados posibilitan establecer parámetros para la planificación, que, junto a especialistas técnicos y asesores, permiten elaborar un plan acorde a las necesidades propias del cantón.

Aunque en el proceso de participación se perciben algunas dificultades (como la falta de capacitación, la politización de las organizaciones sociales y comunitarias en los procesos de organización y participación ciudadana), se busca una mayor colaboración de la comunidad en la planificación territorial. Sus perspectivas, muchas veces, pueden estar limitadas a las decisiones que a nivel ejecutivo y legislativo se presenten, puesto que, en este nivel, finalmente, se aprueban los planes de desarrollo. El director de planificación menciona que: "todas las propuestas son recogidas y analizadas de acuerdo con las necesidades primordiales de los pobladores, pero algunas veces no se contemplan temas sugeridos por los líderes, porque hay otros de mayor interés". Una vez socializada con la comunidad la importancia de sus aportes a la planificación territorial, el organismo técnico del GAD Ibarra depura toda la información y se traslada al PDOT.

Los funcionarios públicos entrevistados destacan como fortalezas el grado de organización social que se ha visto fortalecido en estos últimos años. Señalan que cada vez aumenta el compromiso de participar en estos procesos, reconociendo que la participación ciudadana en la elaboración, ejecución y evaluación de los planes de desarrollo y ordenamiento territorial del cantón es cada vez mayor, debido a que el GAD Ibarra ha mantenido dentro de su política pública dar a conocer la importancia de la participación comunitaria en los diferentes niveles de gestión. Esto se ha hecho mediante la participación de veedurías ciudadanas, observatorios y consejos consultivos, que han proporcionado una adecuada estructura organizacional y acompañamiento técnico, cuyos resultados brindan una oportunidad para que el territorio se desarrolle adecuadamente. Los resultados se pueden observar en la Tabla 4.

\section{Encuesta}

Estos resultados muestran la evaluación de los mecanismos y estrategias con el propósito de lograr diálogos y acuerdos entre el GAD Ibarra y la ciudadanía a través de la realización de encuestas. Si bien el proceso participativo nace desde el GAD, es preciso conocer el grado de participación de los pobladores, promoviendo desde las autoridades el funcionamiento y apoyo al proceso con el cumplimiento oportuno de los acuerdos generados.

Para la aplicación de la encuesta, se consideró a representantes de la ciudadanía, si fuese el caso a líderes comunitarios, con equidad de género (masculino, femenino) de acuerdo al tipo de representatividad, cuyas edades comprendían entre los 30 y 65 años de edad, de las cinco parroquias urbanas y las siete rurales del cantón Ibarra. Para el análisis de los resultados se tomaron en cuenta los más representativos dentro de las encuestas planteadas en el GAD Ibarra, para determinar la participación ciudadana en la planificación y elaboración del PDOT, que se puede interpretar de la siguiente manera:

Con relación a la elaboración de propuestas comunitarias de participación entregadas a las autoridades para que sean tomadas en cuenta en la elaboración de los planes, se observa que la totalidad de los encuestados ha participado, lo que ha permitido verificar que las comunidades se han convertido en partes interesadas y críticas, con un rol importante en el desempeño de la gestión de programas y proyectos. 
Tabla 4. Resultados numéricos y porcentuales de la encuesta

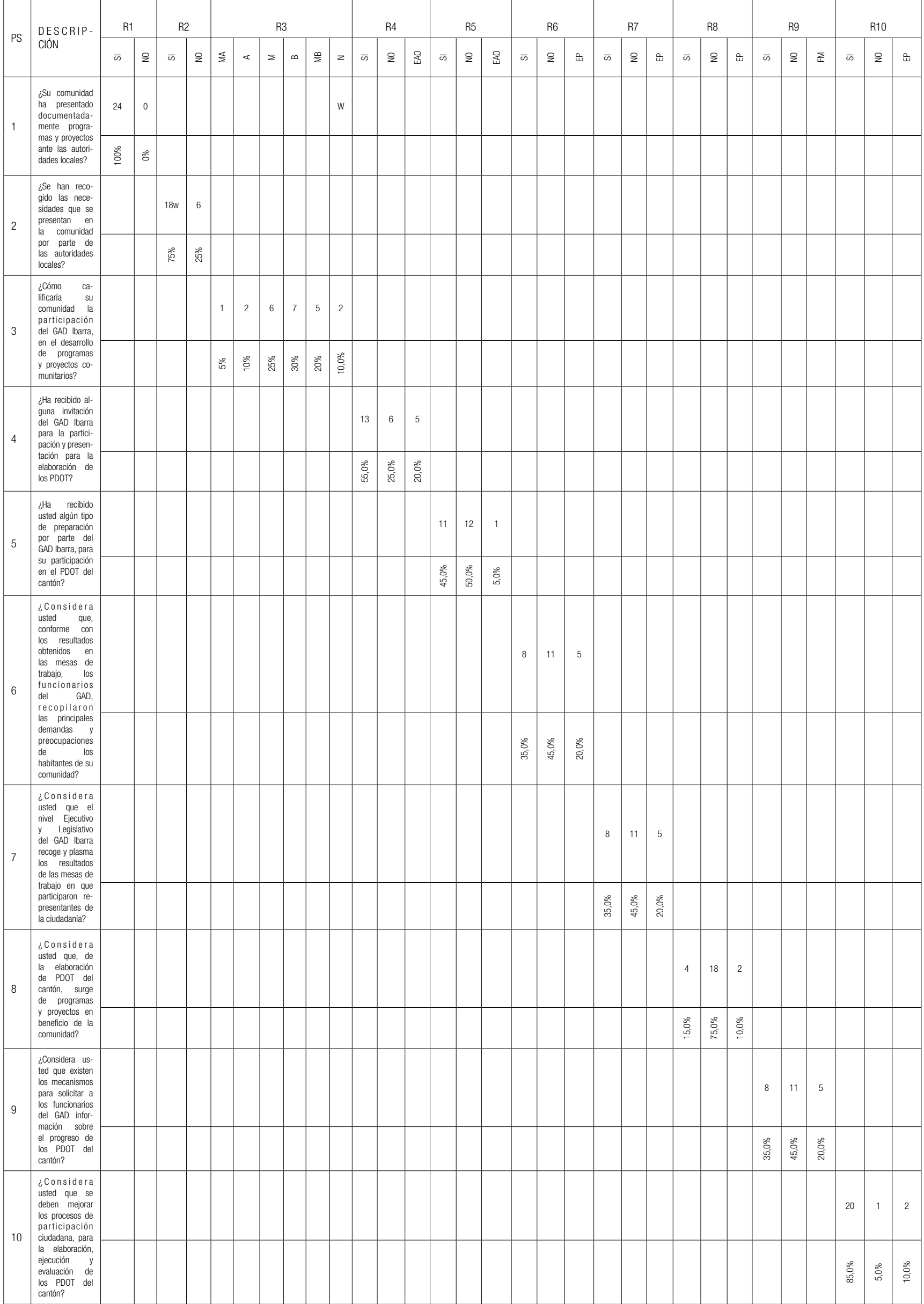

Leyenda: Ps: (preguntas), R (respuesta), MA (muy alto), A (alto), M (medio), B (bajo), MB (muy bajo), N (ninguna), EA0 (en algunas ocasiones), EP (en parte), FM (faltan mecanismos) 
Respecto a si las autoridades locales toman en cuenta las peticiones de la población de acuerdo a sus necesidades, el $75 \%$ considera que si y el $25 \%$ de actores establece que no; se determina que el conocimiento de las necesidades prioritarias ayudará a garantizar la implementación en los planes y programas.

La participación del GAD de Ibarra en la elaboración de programas y proyectos que beneficien a la comunidad, según las encuestas, la ciudadanía tiene la percepción de que no se establecen con claridad las directrices y lineamientos que definen el acompañamiento del GAD en los procesos participativos. Una de las causas principales, es la baja credibilidad de sus líderes, la falta de confianza en la gestión municipal, debido al incumplimiento de las propuestas que, sumado a la deficiente socialización en la elaboración y planificación de los planes, ha provocado falta de interés en el proceso. Los resultados muestran una baja aceptación de participación del GAD en el desarrollo de proyectos con $30 \%$ que los considera bajo, el 30\% muy bajo o ninguno y tan solo el $15 \%$ lo considera alto o muy alto y un $25 \%$ considera participación media.

En la pregunta de si se ha recibido algún tipo de capacitación por parte de los funcionarios del GAD para participar en los planes de desarrollo, los informantes consideran que no se ha realizado ningún taller de capacitación con un $50 \%$, mientras que un $45 \%$ dice que sí y el $5 \%$ en alguna ocasión. Por la importancia que tiene este proceso, las autoridades deben garantizar una preparación adecuada y oportuna que permita alcanzar los objetivos propuestos.

Con relación a las propuestas realizadas por los representantes ciudadanos para que estas sean consideradas y posteriormente desarrolladas por los funcionarios municipales en los PDOTs, se puede identificar que el 35\% consideran que las recogen y plasman en los proyectos, mientras que el $45 \%$ dice que no son tomadas en cuenta las propuestas ciudadanas por las autoridades y el 20\% menciona que "en parte".

Los programas y proyectos se toman como insumos para elaborar los PDOTs en beneficio de las comunidades; en este contexto, el GAD convoca a realizar talleres participativos que tienen la finalidad de tomar en cuenta los aportes ciudadanos mediante la elaboración de guías a considerar para actualizar los planes. En este sentido, se puede señalar que el 75\% de los encuestados indican que los planes surgen de la comunidad mediante mesas de trabajo, mas no del GAD, mientras que el 15\% considera que sí, y un $10 \%$ en parte; por lo que este proceso debe contar con una participación en la mayoría de las organizaciones sociales.

En relación con si se cuenta con los mecanismos para realizar el seguimiento de los PDOTs, si bien es cierto que el GAD debe contar con una estrategia que le permita verificar la implementación, el avance y los resultados a fin de determinar el cumplimiento o no de los programas, los encuestados determinaron que el $45 \%$ consideran que no se aplican estos mecanismos en las etapas de formulación y ejecución del plan, mientras que un $34 \%$ considera que sí, y el $20 \%$ que le faltan estrategias de seguimiento.

En cuanto al mejoramiento de los procesos de participación en la elaboración, seguimiento y evaluación de los PDOTs, es importante resaltar que estos procesos se realizan de acuerdo a la Constitución del Ecuador debiendo establecer los lineamientos para el seguimiento y evaluación de los PD0Ts, que son llevados por el GAD. En este contexto, se establecerán los parámetros de seguimiento, monitoreo y evaluación de los procedimientos a través de la aplicación de mecanismos como el fortalecimiento de organizaciones sociales, asambleas ciudadanas, mesas temáticas, promover las veedurías ciudadanas, con el propósito de garantizar los derechos de participación ciudadana. Por esta razón, el 85\% de los encuestados consideran que se deben mejorar los procesos de participación; por otro lado, no se realizan monitoreos en la etapa de ejecución del plan para evaluar su avance, por lo que el $10 \%$ de encuestados señala que en parte y el $5 \%$ manifiesta que están bien estos procesos.

Bajo las consideraciones anteriores de análisis, se deben mejorar los procesos de participación ciudadana en el GAD Ibarra en la elaboración, ejecución y evaluación de los PDOTs, considerando que la participación ciudadana ha tomado un impulso en los últimos años, el cual brinda la oportunidad para que los diferentes representantes comunitarios y organizaciones puedan participar en los procesos de gestión y decisión a nivel de planificación local y territorial. Es necesario que se revise el sistema de participación con el objetivo de realizar mejoras que beneficien a las partes interesadas.

\section{CONCLUSIONES}

Se debe fortalecer el rol de las organizaciones ciudadanas a través de capacitaciones periódicas que promuevan la concienciación y formación ciudadana en temas de participación ciudadana, presupuestos participativos, planes de desarrollo y ordenamiento territorial y rendición de cuentas, que permitan a los ciudadanos formular diálogos de forma crítica en el cumplimiento de procesos participativos, frente a las necesidades reales de la comunidad.

Con respecto al proceso participativo del GAD Ibarra, se encuentra regulado en la ordenanza municipal, pero el establecimiento de estas normas no garantiza la participación; por otro lado, se observa que no existen suficientes espacios y mecanismos que fomenten la participación y control social mediante las veedurías para realizar el monitoreo y seguimiento y la evaluación del proceso.

El GAD Ibarra debe promover un diálogo permanente que permita lograr una discusión mucho más abierta con los actores locales en temas de interés general y del territorio en los planes de desarrollo local, aceptando distintas posturas y escalas de discusión, suscitando que los movimientos sociales pasivos a la problemática participativa se inserten efectivamente en el proceso.

Las propuestas se realizan en torno a los hallazgos encontrados y que se evidencian en la falta de comunicación e información de los representantes de la comunidad en cuanto a su participación en los proyectos de ordenamiento y desarrollo territorial.

Por último, el desafío más urgente para el GAD del cantón Ibarra, a juicio de los autores, es construir una sociedad motivada por la participación. Los requisitos de la democracia se cumplen cuando las oportunidades de participación ciudadana están presentes, independientemente de si el ciudadano participa o no.

Contribución de los autores: el artículo en sus secciones de resumen, introducción, métodos, resultados y discusión, conclusiones y bibliografía se llevó a cabo de forma conjunta por los dos autores.

Financiamiento y originalidad: Esta investigación no recibió financiamiento externo, este artículo es original en su totalidad tomando en cuenta que no ha sido publicado o postulado previamente en ningún órgano editorial, ni tampoco ha participado en ningún encuentro científico; ninguna de las partes de este articulo ha sido utilizado para ningún tipo de comunicación en ponencias y seminarios. 


\section{REFERENCIAS}

Acosta, A. (2017). Breve historia económica del Ecuador. Quito: Corporación Editora Nacional.

Albert, M. (2013). La Investigación Educativa. Claves Teóricas. España: Mc Graw Hill.

Álvarez, C., \& Sierra, V. (2012). Metodología de la Investigación Científica. Cochabamba: Grupo Editorial Kipus.

Calvo, M., Ger, A., Gómez, J., Martínez, M., \& Tomás, E. (2017). Evaluación de participación ciudadana en América Latina. Washington: OEA.

GAD Ibarra. (2017). Plan de Desarrollo y Ordenamiento Territorial del Cantón Ibarra. Ibarra: GAD Ibarra.

GAD, Ibarra. (2011). Ordenaza que reglamenta el Sistema de Participación Ciudadana en el Gobierno Autónomo Descentralizado de Ibarra. Ibarra: GAD Ibarra.

Guerrero Quimbiulco, G. D. (2019). Partipación ciudadana en los planes de desarrollo y ordenamiento territorial, ciudad ibarra, Ecuador. Trabajo Final de Máster, Universidad de Barcelona: UNIBA, Barcelona.

Guillen, A., Sáenz, K., Badii, M., \& Castillo, J. (2018). Origen, espacio y niveles de participación ciudadanan en Ecuador. International Journal of Good Conscience, 50-61.

Hernández, R., Fernández, C., \& Baptista, P. (2014). Metodología de la investigación (Sexta Edición ed.). México: Editorial Mc Graw Hill.

SENPLADES. (2017). Encuesta Nacional de Participación Ciudadana INEC-SENPLADES. Quito: SENPLADES.

SENPLADES. (2018). Guía para la formulación participativa en los PD y OT. Quito: SENPLADES.

Veneziano, A. (2017). El presupuesto participativo en Montevideo: un caso pionero en América Latina. Revista Social Cambia, Vol. 39., 124-146. 